\title{
The risk of upper gastrointestinal bleeding in patients treated with hemodialysis: a population-based cohort study
}

\author{
Chien-Chun Kuo ${ }^{1}$, Hsin-Wei Kuo ${ }^{2}$, I-Ming Lee ${ }^{3}$, Chien-Te Lee ${ }^{1}$ and Chun-Yuh Yang ${ }^{3,4^{*}}$
}

\begin{abstract}
Background: There are no prior studies that have estimated the risk of upper gastrointestinal bleeding (UGIB) among the dialysis population relative to the general population. The aim of this study was to examine the risk of UGIB among end-stage renal disease (ESRD) patients during a 6-year period following their initiation of hemodialysis (HD) therapy in Taiwan- a country with the highest incidence of ESRD in the world, using general population as an external comparison group.

Methods: Data were obtained from the Taiwan National health Insurance Research Database. In total, 796 patients who were beginning HD between 1999 and 2003 were recruited as the study cohort and 3,184 patients matched for age and sex were included as comparison cohort. Multivariate Cox proportional hazard regression models were used to adjust for confounding and to compare the 6-year UGIB-free survival rate between these two cohorts.

Results: The incidence rate of UGIB (42.01 per 1000 person-year) was significantly higher in the HD cohort than in the control cohort (27.39 per 1000 person-years). After adjusting for potential confounders, the adjusted hazard ratios for UGIB during the 6-year follow-up periods for HD patients was 1.27 (95\% Cl=1.03-1.57) compared to patients in the comparison cohort.
\end{abstract}

Conclusions: We conclude that HD patients were at an increased risk for UGIB compared with the general population.

Keywords: Upper gastrointestinal bleeding, End-stage renal disease, Cohort study

\section{Background}

The increasing number of patients with end-stage renal disease (ESRD) requiring dialysis therapy is becoming a worldwide public health problem and puts a substantial burden on health care resources. Taiwan is also confronting this severe challenge [1]. The incidence and prevalence of ESRD in Taiwan were not only the highest of some 30 countries reported by USRDS, but were $2-4$ times higher than most European countries [2]. There have been 2.6- and 3.7-fold increases in incidence and prevalence during the past decade in Taiwan, respectively [1]. The financial burden of dialysis has exceeded

\footnotetext{
* Correspondence: chunyuh@kmu.edu.tw

${ }^{3}$ Department of Public Health, Kaohsiung Medical University, 100 Shih-Chuan 1st RD, Kaohsiung 80708, Taiwan

${ }^{4}$ Division of Environmental Health and Occupational Medicine, National Health Research Institute, Miaoli, Taiwan

Full list of author information is available at the end of the article
}

the expenditure on all cancers combined, and reducing the number of people with ESRD constitutes a national health priority in Taiwan [3].

Studies suggest that acute gastrointestinal hemorrhage, especially upper gastrointestinal bleeding (UGIB), is the most frequent bleeding complication of acute renal failure (ARF), even though the reported incidence ranges widely [4]. Reports also suggest that the prevalence or incidence of UGIB in patients with ESRD may be greater than that in the general population [5-10]. It has been estimated that UGIB accounts for 3 to $7 \%$ of all deaths among ESRD patients [5]. However, some studies found that patients with chronic renal failure are not at risk for developing chronic peptic ulcers [11,12]. Most of the previous studies were conducted in western countries $[5,6,8,10-12]$ and were done before the era of extensive 
use of pharmacological prophylaxis of upper gastrointestinal stress lesions in critically ill patients [4].

To date, there are limited data regarding the occurrence of UGIB in dialysis patients. Most of the above-mentioned epidemiologic studies suffer from methodological limitations such as a relatively small size, case series without controls or not taking potential confounders into considerations in the regression model. With these methodological issues in mind, we conducted a cohort study using general population as an external comparison group to determine the risk of UGIB among ESRD patients during a 6-year period following their initiation of hemodialysis (HD) therapy in Taiwan- a country with the highest incidence of ESRD in the world. To our knowledge, this is the first population-based cohort study to investigate the relationship between HD and the risk of UGIB.

\section{Methods}

\section{Data source}

The National Health Insurance (NHI) program, which provides compulsory universal health insurance, was implemented in Taiwan on March 1, 1995. Under the NHI, 98\% of the island's population receives all forms of health care services including outpatient services, inpatient care, Chinese medicine, dental care, childbirth, physical therapy, preventive health care, home care, and rehabilitation for chronic mental illness. In cooperation with the Bureau of NHI, the National Health Research Institute (NHRI) of Taiwan randomly sampled a representative database of $1,000,000$ subjects from the entire NHI enrollees by means of a systematic sampling method for research purposes. There were no statistically significant differences in age, gender, and healthcare costs between the sample group and all enrollees, as reported by the NHRI. This dataset (from January 1996 to December 2009) includes all claim data for these 1,000,000 subjects, offers a good opportunity to examine the risk of UGIB occurring among patients with ESRD. These databases have previously been used for epidemiological research, and information on prescription use, diagnoses, and hospitalizations has been shown to be of high quality [1,13-15].

Because the identification numbers of all individuals in the NHRI databases were encrypted to protect the privacy of the individuals, this study was exempt from full review by the Kaohsiung Medical University Institution Review Board.

\section{Study cohorts}

From this database, we selected all patients who were beginning chronic HD or peritoneal dialysis between January 1, 1999 and December 31, 2003 and who have survived more than 90 days of renal replacement therapy for ESRD $(n=1046)$ [16]. We excluded individuals younger than 18 years of age because they were not at appreciable risk of
UGIB and in order to limit the study subjects to an adult population $(n=9)$. ESRD patients are defined as those who had catastrophic illness registration cards for ESRD (ICD-9-CM code 585) and started renal replacement therapy. In Taiwan, patients who reached ESRD with the need for long-term renal replacement therapy can apply for catastrophic illness registration cards given by the Bureau of Health Insurance. These patients do not need to pay copayments when they seek health care for renal disease. A detailed description of this cohort is published elsewhere [17]. We excluded subjects with any type of UGIB diagnosed before or within 90 days of their index ambulatory care visit (the date of a patient's initiating dialysis) $(n=169)$. In addition, we also excluded those subjects who received a renal transplant $(\mathrm{n}=3)$ or peritoneal dialysis $(n=57)$. A total of 808 incident HD patients were identified between January 1, 1999 and December 31, 2003.

The comparison cohort were selected from the remaining patients in the database. We first excluded patients who had been diagnosed with chronic kidney disease (ICD-9 CM codes 250.4", 274.1", 283.11, 403."1, 404." 2, 404."3, 440.1, 442.1, 447.3, 572.4, 580-588, 642.1", 646.2") [1] during the period 1996-2009 and those younger than 18 years of age. For each study cohort patient, 4 reference subjects were identified randomly and matched for gender, age (birth of year), and the year of index ambulatory care visit. For the comparison cohort, the index ambulatory care visit was their first ambulatory care visit occurring in the index year. In addition, we excluded patients who had been diagnosed with UGIB before or within 90 days of their index ambulatory care visits. Of the 808 incident HD cases ascertained, no controls could be found for 12 of the cases. A total of 3,184 subjects served as a comparison cohort group.

\section{Incidence of UGIB}

Patients were considered to have experienced UGIB if a hospital discharge diagnosis indicating UGIB, as defined by any one of the gastric, duodenal, peptic, and gastrojejunal bleeds were reported (ICD-9-CM codes 531.0X, 531.2X, 531.4X, 531.6X, 532.00, 532.2X, 532.4X, 532.6X, 533.0X, 533.2X, 533.4X, 533.6X, 534.0X, 534.2X, 534.4X, 534.6X, 535.X1). Gastrointestinal bleed, unspecified, was not used to identify UGIB because it was felt to be too nonspecific with regard to location of bleeding.

\section{Potential confounders}

For all individuals in both cohorts, we obtained data on potential confounders which are documented risk factors for UGIB, including hypertension (ICD-9-CM, codes 401-405), diabetes (ICD-9-CM, code 250), hyperlipidemia (ICD-9-CM, code 272), coronary heart disease (ICD-9-CM, codes 410-414), chronic liver disease and cirrhosis (ICD-9-CM, code 571), and Helicobacter pylori 
(HP) eradication, recorded during 12 months before the index ambulatory care visit. In addition, we also obtained prescription data for medications for aspirin, non-steroidal antiinflammatory drugs (NSAIDs), $\mathrm{H}_{2}$ receptor antagonists $\left(\mathrm{H}_{2} \mathrm{RA}\right)$, and proton pump inhibitor (PPI) use, recorded between the index date to the date of UGIB hospitalization, death or the end of the study. We collected the date of prescription, the daily dose, and the number of days supplied. The defined daily doses (DDDs) recommended by the WHO were used to quantify the usage of medications [18].

\section{Statistics}

For comparisons of proportions between the study and comparison cohorts, the chi-square test was used. The mean doses of medication use (aspirin, NSAIDs, $\mathrm{H}_{2} \mathrm{RA}$, and PPI) were calculated and compared between the study and comparison cohorts using paired t test; because of the skew distribution of these medication use data, medication use data were log-transformed to improve normality. For the analysis, the subjects were categorized into one of the three exposure categorizes: nonusers (subjects with no prescription at any time between the index date and the date of UGIB hospitalization, death or the end of the study), and users of doses equal to or below the mean, and users of doses above the mean based on the distribution of use among controls. Each patient was individually tracked for a 6 -year period starting from the index ambulatory care visit to identify whether the patient had experienced UGIB during the follow-up period. The person-years of follow-up were calculated for each patient from the date of the index ambulatory care visit to the date of UGIB hospitalization, death or the end of the study (a 6-year follow-up period), whichever occurred first. Incidence rates were calculated by dividing the number of UGIB hospitalizations by the total person-years of follow-up. Kaplan-Meier curves and log-rank tests were used to explore the difference in the risk of developing UGIB between the 2 cohorts. A multivariate frailty Cox proportional hazards regression model, which incorporates an unmeasured "random" effect (the frailty) in the hazard function, was used to estimate the hazard ratios (HR) and 95\% confidence intervals (CI) adjusted for the above-mentioned potential confounders [19]. The assumption of proportional hazards was assessed by including an interaction term between time and exposure (HD patients vs patients in the comparison cohort) in the model, and the proportional assumption was satisfied. Analyses were performed using the SAS statistical package (version 9.2, SAS Institute Inc., Cary, NC, USA). All statistical tests were two-sided. Values of $\mathrm{p}<0.05$ were considered statistically significant.

\section{Results}

There were 796 patients in our HD cohort that were compared with 3,184 selected matched controls. Table 1 presents the distribution of demographic characteristics and selected medical conditions of the study subjects. Of the 3,980 patients sampled, the mean age was 56.72 years $(\mathrm{SD}=13.74)$, with means of $56.72(\mathrm{SD}=13.75)$ and 56.72 $(\mathrm{SD}=13.74)$ for $\mathrm{HD}$ patients and comparison patients, respectively. HD patients had a significant higher rate of hypertension, diabetes, hyperlipidemia, coronary heart disease, and liver cirrhosis. HD patients were also significantly more likely to have PPI use.

Table 1 Basic characteristics for hemodialysis patients versus the comparison patients

\begin{tabular}{|c|c|c|c|c|c|}
\hline \multirow[t]{2}{*}{ Variable } & \multicolumn{2}{|c|}{$\begin{array}{c}\text { HD } \\
\text { patients } \\
n=796\end{array}$} & \multicolumn{2}{|c|}{$\begin{array}{c}\text { Comparison } \\
\text { patients } \\
n=3184\end{array}$} & \multirow[t]{2}{*}{$p$ value } \\
\hline & $\mathbf{n}$ & $\%$ & $\mathrm{n}$ & $\%$ & \\
\hline \multicolumn{6}{|l|}{ Gender } \\
\hline Male & 365 & 45.85 & 1460 & 45.85 & - \\
\hline Female & 431 & 54.15 & 1724 & 54.15 & \\
\hline Age (mean $\pm S D)$ & \multicolumn{2}{|c|}{$56.72 \pm 13.75$} & \multicolumn{2}{|c|}{$56.72 \pm 13.74$} & - \\
\hline$<50$ & 241 & 30.28 & 964 & 30.28 & 0.99 \\
\hline $50-64$ & 296 & 37.19 & 1184 & 37.19 & \\
\hline $65-74$ & 200 & 25.13 & 798 & 25.06 & \\
\hline$>74$ & 59 & 7.41 & 238 & 7.47 & \\
\hline \multicolumn{6}{|l|}{ Hypertension } \\
\hline Yes & 566 & 71.11 & 1032 & 32.41 & $<0.001$ \\
\hline No & 230 & 28.89 & 2152 & 67.59 & \\
\hline \multicolumn{6}{|l|}{ Diabetes } \\
\hline Yes & 288 & 36.18 & 395 & 12.41 & $<0.001$ \\
\hline No & 508 & 63.82 & 2789 & 87.59 & \\
\hline \multicolumn{6}{|l|}{ Coronary heart disease } \\
\hline Yes & 162 & 20.35 & 372 & 11.68 & $<0.001$ \\
\hline No & 634 & 79.65 & 2812 & 88.32 & \\
\hline \multicolumn{6}{|l|}{ Hyperlipidemia } \\
\hline Yes & 145 & 18.22 & 446 & 14.01 & 0.003 \\
\hline No & 651 & 81.78 & 2738 & 85.99 & \\
\hline \multicolumn{6}{|l|}{ Liver cirrhosis } \\
\hline Yes & 20 & 2.51 & 33 & 1.04 & 0.001 \\
\hline No & 776 & 97.49 & 3151 & 98.96 & \\
\hline \multicolumn{6}{|l|}{ HP eradication } \\
\hline Yes & 54 & 6.78 & 201 & 6.31 & 0.627 \\
\hline No & 776 & 97.49 & 3151 & 98.96 & \\
\hline Aspirin use (gm/person-year) & \multicolumn{2}{|c|}{$11.28 \pm 35.76$} & \multicolumn{2}{|c|}{$13.05 \pm 47.29$} & 0.247 \\
\hline NSAIDs use (DDDs/person-year) & \multicolumn{2}{|c|}{$14.72 \pm 29.54$} & \multicolumn{2}{|c|}{$41.99 \pm 75.14$} & $<0.001$ \\
\hline PPI use (DDDs/person-year) & \multicolumn{2}{|c|}{$16.15 \pm 184.80$} & \multicolumn{2}{|c|}{$3.02 \pm 16.39$} & $<0.001$ \\
\hline $\mathrm{H}_{2} \mathrm{RAs}$ use (DDDs/person-year) & \multicolumn{2}{|c|}{$6.97 \pm 21.30$} & \multicolumn{2}{|c|}{$5.60 \pm 18.44$} & 0.490 \\
\hline
\end{tabular}


Table 2 Crude hazard ratios of UGIB in hemodialysis cohort compared to patients in comparison cohort during the 6-year follow-up period

\begin{tabular}{|c|c|c|}
\hline & $\begin{array}{c}\mathrm{HD} \\
\text { patients } \\
(\mathrm{n}=796)\end{array}$ & $\begin{array}{c}\text { Comparison } \\
\text { patients } \\
(n=3184)\end{array}$ \\
\hline No. of UGIB & 176 & 471 \\
\hline Person time (years) & 4189.06 & 17196.73 \\
\hline Incidence ${ }^{a}$ & 42.01 & 27.39 \\
\hline Crude HR (95\% Cl) & $\begin{array}{c}1.50 \\
(1.25-1.80)\end{array}$ & 1.00 \\
\hline No. of gastric ulcer & 68 & 144 \\
\hline Person time (years) & 4189.06 & 17196.73 \\
\hline Incidence $^{a}$ & 16.23 & 8.37 \\
\hline Crude HR (95\% Cl) & $\begin{array}{c}1.86 \\
(1.37-2.51)\end{array}$ & 1.00 \\
\hline No. of duodenal ulcer & 26 & 86 \\
\hline Person time (years) & 4189.06 & 17196.73 \\
\hline Incidence ${ }^{a}$ & 6.21 & 5.00 \\
\hline Crude HR (95\% Cl) & $\begin{array}{c}1.21 \\
(0.77-1.90)\end{array}$ & 1.00 \\
\hline No. of peptic ulcer & 50 & 160 \\
\hline Person time (years) & 4189.06 & 17196.73 \\
\hline Incidence $^{a}$ & 11.94 & 9.30 \\
\hline Crude HR (95\% Cl) & $\begin{array}{c}1.24 \\
(0.89-1.72)\end{array}$ & 1.00 \\
\hline No. of gastrojejunal ulcer & 2 & 10 \\
\hline Person time (years) & 4189.06 & 17196.73 \\
\hline Incidence ${ }^{a}$ & 0.48 & 0.58 \\
\hline Crude HR (95\% Cl) & $\begin{array}{c}0.94 \\
(0.20-4.44)\end{array}$ & 1.00 \\
\hline No. of gastritis/duodenitis with bleeding & 30 & 71 \\
\hline Person time (years) & 4189.06 & 17196.73 \\
\hline Incidence ${ }^{a}$ & 7.16 & 4.13 \\
\hline Crude HR (95\% Cl) & $\begin{array}{c}1.74 \\
(1.12-2.70)\end{array}$ & 1.00 \\
\hline
\end{tabular}

a per 1000 person-years.

Table 2 shows the distribution of UGIB during the 6-year follow-up periods for these two cohorts. Of the total 3,980 sampled patients, 647 patients (16.26\%) developed UGIB throughout the study periods, 176 (22.11\% of the HD patients) from the study cohort (with an incidence rate of 42.01 per 1,000 person-years) and 471 (14.79\%) from the comparison cohort (with an incidence rate of 27.39 per 1,000 person-years). Gastric ulcers were found to be the most common source of bleeding (16.23 per 1000 person-years), while bleeding resulting from a gastrojejunal ulcer was least frequent (0.48 per 1000 person-years) among HD patients.

After adjusting for age, gender, hypertension, diabetes, hyperlipidemia, coronary heart disease, liver cirrhosis,
Table 3 Adjusted hazard ratio (HRs) for UGIB among the sampled patients identified by Cox regression analysis

\begin{tabular}{lc}
\hline Variable & Adjusted HR (95\% Cl) \\
\hline Patients receiving HD & $1.27(1.03-1.57)$ \\
Hypertension & $1.13(0.91-1.39)$ \\
Diabetes & $1.09(0.86-1.39)$ \\
Coronary heart disease disease & $1.14(0.88-1.49)$ \\
Hyperlipidemia & $1.14(0.88-1.46)$ \\
Liver cirrhosis & $1.14(0.59-2.20)$ \\
Medication use & \\
$\quad$ NSAIDs (per 1 DDD increase) & $1.00(0.99-1.00)$ \\
$\quad$ PPI (per 1 DDD increase) & $1.01(1.00-1.01)$ \\
\hline${ }^{a}$ Adjusted for all covariates listed.
\end{tabular}

the use of NSAIDs, and PPI, Cox multivariate hazard regression analysis showed that the hazard of UGIB during the 6-year follow-up periods was 1.27 times greater (95\% CI $=1.03-1.57)$ for patients treated with hemodialysis than for the comparison cohort (Table 3; Figure 1).

\section{Discussion}

The only study estimating the incidence of UGIB in dialysis patients is performed in the United States, which reported an incidence rate of 22.77 per 1000 personyears [10]. The incidence of UGIB was 42.01 per 1000 person-years in the present study. The reason for a higher incidence rate of UGIB in Taiwanese HD patients are not clear. It may be that renal transplant recipients and peritoneal dialysis patients were excluded in the present study and that renal transplant recipients and peritoneal dialysis patients had a lower risk of UGIB compared to HD patients [10]. However, the possibility that the incidence rates of UGIB in Taiwanese population are higher than that of the United States and that characteristics of HD patients are substantially different

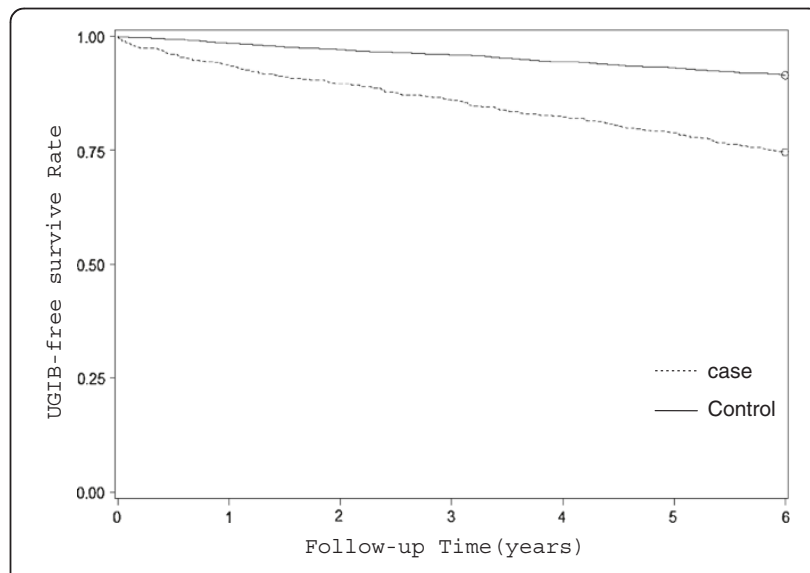

Figure 1 UGIB-free survive rates for HD patients and patients in the comparison cohort. 
from that in the United States can not be excluded. Further study is needed to clarify the reason.

There are no prior studies that have estimated the risk of UGIB among the dialysis population relative to the general population. Previous study has only used dialysis patients as a cohort to identify patient characteristics associated with the incident UGIB without seeking a comparison group [10]. Because of the uniqueness of the exposure (hemodialysis), special exposure cohort (HD patients) provides a statistically efficient way of studying this factor, which would be rare in general cohort. Special exposure cohort (dialysis patients), however, require an external comparison group. To the best of our knowledge, this study is among the first matched cohort study to examine the risk of UGIB among HD patients using general population as an external comparison group (with a age- and gender-matched comparison group). In this population-based cohort study, after adjusting for potential confounders, we found that HD patients were 1.27 times more likely than the general population to experience UGIB during the 6-year follow-up period. Our findings support previous studies that found chronic renal failure can be a UGIB risk factor.

The major strength of our study is the use of a computerized database, which is population based and is highly representative and allows a clear observation of the temporal relationship between HD and UGIB. Because we included a national sample of ESRD patients initiating HD therapy between January 1, 1999 and December 31, 2003, and because the control subjects in this study were selected from a simple random sampling of insured general population, we can rule out the possibility of selection bias. Moreover, the large sample size affords considerable statistical power for detecting real difference between the two cohorts.

Several limitations of the present study should be noted. First, diagnoses of ESRD, UGIB, or any other comorbid medical conditions and prescription information rely on administrative claims data may be less accurate than those obtained according to standardized criteria and misclassification is possible. However, from previous studies, the NHIRD is of acceptable quality to provide reasonable estimations for epidemiological data [13-15]. Moreover, regular chart-review and cross-checking mechanisms conducted by Taiwan's NHI Bureau do facilitate the accuracy of coding [20]. Second, although we adjusted for several potential confounders in the statistical analysis, a number of possible confounding variables, including smoking [21], alcohol use [22], physical inactivity [21,23], and Helicobacter pylori infection [24], which might be associated with UGIB development were not included in our database. These unmeasured risk factors might have biased results if they were differentially associated with case versus comparison cohort. Third, we were not able to contact the patients directly about their use of medications because of anonymization of their identification number. Using pharmacy records representing dispensing data rather than usage data might have introduced an overestimation of medication use. Fourth, the case cohort includes only HD patients. It has been hypothesized that intermittent anticoagulation may place HD patients at greater risk for UGIB. If this were the case, one would anticipate that peritoneal dialysis and renal transplantation would be associated with a lower risk for UGIB compared to HD [10]. Therefore, the results of this study may not be generalizable to patients receiving peritoneal dialysis or renal transplantation.

\section{Conclusion}

In summary, our study results provide evidence that HD patients are at an increased risk for UGIB compared with general population. Therefore, prevention of UGIB is becoming an important issue in the maintenance of good quality of life for HD patients.

\section{Competing interests}

All authors have no conflict of interests; This paper is neither the entire paper nor any part of its content has been published or accepted elsewhere.

\section{Authors' contributions}

KCC did the statistical analysis and wrote the manuscript. KHW, LIM and LCT provided essential insight into the interpretation of the results. YCY contributed to study design and interpretation of the data. All authors read and approved the final manuscript.

\section{Acknowledgments}

This study is based in part on data from the National Health Insurance Research Database provided by the Bureau of National Health Insurance, Department of Health and managed by National Health Research Institutes. The interpretation and conclusions contained herein do not represent those of Bureau of National Health Insurance, Department of Health or National Health Research Institutes.

This study was supported in part by a grant from the National Science Council, Executive Yuan, Taiwan (NSC-100-2314-B-037-023-MY2).

\section{Author details}

${ }^{1}$ Division of Nephrology, Department of Internal Medicine, Kaohsiung ChangGung Memorial Hospital and Chang-Gung University College of Medicine, Kaohsiung, Taiwan. ${ }^{2}$ Division of Nephrology, Department of Internal Medicine, Yuan's General Hospital, Kaohsiung, Taiwan. ${ }^{3}$ Department of Public Health, Kaohsiung Medical University, 100 Shih-Chuan 1st RD, Kaohsiung 80708, Taiwan. ${ }^{4}$ Division of Environmental Health and Occupational Medicine, National Health Research Institute, Miaoli, Taiwan.

Received: 1 July 2012 Accepted: 14 January 2013

Published: 16 January 2013

\section{References}

1. Kuo HW, Tsai SS, Tiao MM, Yang CY: Epidemiologic features of CKD in Taiwan. Am J Kidney Dis 2007, 49:46-55.

2. United States Renal Data System: USRDS 2007 Annual Data Report. The National Institutes of Health, National Institutes of Diabetes and Digestive and Kidney Diseases. Bethesda, MD: United States Renal Data System; 2007.

3. Wen CP, Cheng TY, Tsai MK, et al: All-cause mortality attributable to chronic kidney disease: a prospective cohort study based on 462293 adults in Taiwan. Lancet 2008, 371:2173-2182.

4. Fiaccadori E, Maggiore U, Clima B, Melfa L, Rotelli C, Borghetti A: Incidence, risk factors, and prognosis of gastrointestinal hemorrhage complicating acute renal failure. Kidney Int 2001, 59:1510-1519. 
5. Posner GL, Fink SM, Huded FV, Dunn I, Calderone PG, Joglekar SS: Endoscopic findings in chronic hemodialysis patients with upper gastrointestinal bleeding. Am J Gastroenterol 1983, 78:720-721.

6. Boyle JM, Johnston B: Acute upper gastrointestinal hemorrhage in patients with chronic renal disease. Am J Med 1983, 75:409-412.

7. Gheissari A, Rajyaguru V, Kumashiro R, Matsumoto T: Gastrointestinal hemorrhage in end stage renal disease patients. Int Surg 1990, 75:93-95.

8. Alvarez L, Puleo J, Balint JA: Investigation of gastrointestinal bleeding in patients with end stage renal disease. Am J Gastroenterol 1993, 88:30-33.

9. Tsai $\mathrm{CJ}$, Hwang JC: Investigation of upper gastrointestinal hemorrhage in chronic renal failure. J Clin Gastroenterol 1996, 22:2-5.

10. Wasse $H$, Gillen DL, Ball AM, et al: Risk factors for upper gastrointestinal bleeding among end-stage renal disease patients. Kidney Int 2003, 64:1455-1461

11. Andriulli A, Malfi B, Recchia S, Ponti V, Triolo G, Segoloni G: Patients with chronic renal failure are not at a risk of developing chronic peptic ulcers. Clin Nephrol 1985, 23:245-248.

12. Kang JY, Wu AY, Sutherland IH, Vathsala A: Prevalence of peptic ulcer in patients undergoing maintenance hemodialysis. Dig Dis Sci 1988, 33:774-778

13. Chiu HF, Huang YW, Chang CC, Yang CY: Use of proton pump inhibitors increased the risk of hip fracture: a population-based case-control study. Pharmacolepidemiol Drug Saf 2010, 11:1131-1136.

14. Tiao MM, Tsai SS, Kuo HW, Chen CL, Yang CY: Epidemiological features of biliary atresia in Taiwan, a national study 1996-2003. J Gastroenterol Hepatol 2008, 23:62-66.

15. Wu CY, Kuo KN, Wu MS, Chen YJ, Wang CB, Lin JT: Early helicobacter pylori eradication decreases risk of gastric cancer in patients with peptic ulcer disease. Gastroenterology 2009, 137:1641-1648.

16. United States Renal Data System: Researcher's Guide to the USRDS Database, National Institutes of Health, National Institutes of Diabetes and Digestive and Kidney Diseases. Bethesda, MD: United States Renal Data System; 1999.

17. Kuo CC, Lee CT, Lee IM, Ho SC, Yang CY: The risk of herpes zoster in patients treated with long-term hemodialysis: a matched cohort study. Am J Kid Dis 2012, 59:428-433.

18. WHO Collaborating Center for Drugs Statistics Methodology. ATC index with DDDs 2003. Oslo: WHO; 2003

19. Hosmer DW, Lemeshow S: Applied survival analysis. New York: John Wiley \& Sons, Inc; 1999:317-326.

20. Lin HC, Chien CW, Ho JD: Herpes zoster ophthalmicus and the risk of stroke. Neurology 2010, 74:792-797.

21. Kaplan RC, Heckbert SR, Psaty BM: Risk factors for hospitalized upper or lower gastrointestinal tract bleeding in treated hypertensives. Prev Med 2002, 34:455-462.

22. Pahor M, Guralnik JM, Salive ME, Chrischilles EA, Manto A, Wallace RB: Disability and severe gastrointestinal hemorrhage: a prospective study of community-dwelling older persons. J Am Geriatr Soc 1994, 42:816-825

23. Kaplan RC, Heckbert SR, Koepsell TD, et al: Risk factors for hospitalized gastrointestinal bleeding among older persons. J Am Geriatr Soc 2001, 49:126-133.

24. Vergara M, Catalan M, Gisbert JP, Calvet X: Meta-analysis: role of helicobacter pylori eradication in the prevention of peptic ulcer in NSAID users. Aliment Pharmacol Ther 2005, 21:1411-1418.

doi:10.1186/1471-2369-14-15

Cite this article as: Kuo et al:: The risk of upper gastrointestinal bleeding in patients treated with hemodialysis: a population-based cohort study. BMC Nephrology 2013 14:15.

\section{Submit your next manuscript to BioMed Central and take full advantage of:}

- Convenient online submission

- Thorough peer review

- No space constraints or color figure charges

- Immediate publication on acceptance

- Inclusion in PubMed, CAS, Scopus and Google Scholar

- Research which is freely available for redistribution

Submit your manuscript at www.biomedcentral.com/submit 